\title{
Inhaled steroids for asthma after emergency department discharge
}

\section{John Hillenkamp ${ }^{1}$ (1) Allan Wolfson ${ }^{2}$ (1)}

${ }^{1}$ Corresponding author. University of Pittsburgh (Pittsburgh). Pennsylvania, United States of America. hillenkampjz@upmc.edu ${ }^{2}$ University of Pittsburgh (Pittsburgh). Pennsylvania, United States of America. wolfsonab@upmc.edu

\begin{tabular}{|l|l|}
\hline NNT Color Recommendation & Yellow (Unclear if benefits) \\
\hline Summary Heading & $\begin{array}{l}\text { Inhaled steroids do not reduce asthma relapse rate when } \\
\text { added to oral corticosteroids. However, given alone, they } \\
\text { may be as effective as oral corticosteroids but current data } \\
\text { are limited. }\end{array}$ \\
\hline Benefits in NNT & No one was helped \\
\hline Benefits in percentages & No one was helped \\
\hline Harms in NNT & No one was harmed \\
\hline Harms in percentages & No one was harmed \\
\hline Efficacy Endpoints & Asthma relapse \\
\hline Harm Endpoints & $\begin{array}{l}\text { Side effects (headache, infection, nervousness, tremors, } \\
\text { thrush, nausea, dyspepsia, insomnia, dysphonia, } \\
\text { palpitations) }\end{array}$ \\
\hline Who was in the studies & $\begin{array}{l}2205 \text { adult patients presenting to the emergency } \\
\text { department with mild to moderate acute asthma. }\end{array}$ \\
\hline
\end{tabular}

Source: Edmonds ML, Milan SJ, Brenner BE, Camargo CA Jr, Rowe BH. Inhaled steroids for acute asthma following emergency department discharge. Cochrane Database Syst Rev. 2012;12:CD002316. doi: 10.1002/14651858.CD002316.pub2

\section{Narrative}

Asthma is one of the most common chronic diseases in the world and currently affects an estimated 300 million people ${ }^{1}$. Asthma is a chronic inflammatory disease of the lungs that leads to airway edema, airway obstruction, and bronchospasm. One in every 250 deaths worldwide can be attributed to asthma'. An asthma attack is an acute worsening of asthmatic symptoms such as wheezing, shortness of breath, chest tightness, and coughing. Patients who experience an acute asthma attack and are treated in the emergency department often require multi-modal therapy, including treatment with inhaled beta-2 agonists and systemic corticosteroids. Relapse of symptoms after an asthma exacerbation is common, prompting many patients to return to the emergency department. Currently, short-term oral corticosteroids are commonly used to prevent relapse after discharge ${ }^{2}$. Inhaled corticosteroids have been used as long-term control treatment for asthma in many patients and work through anti-inflammatory effects 3 . It is thought that the use of inhaled corticosteroids upon discharge from the emergency department may also reduce the risk of asthma relapse. 
This updated Cochrane systematic review ${ }^{4}$ expands on previously published data that demonstrated no beneficial effect on relapse rate when inhaled corticosteroids were added to treatment with systemic corticosteroids ${ }^{5}$. The review included 12 trials and a total of 2205 patients. Two separate analyses were performed: one for inhaled corticosteroids plus systemic steroids versus systemic therapy alone, and the other for inhaled corticosteroids alone versus systemic therapy alone.

The first analysis included three trials with a total of 909 patients. It found that the addition of inhaled corticosteroids to systemic corticosteroid treatment offered no benefit in terms of relapse rate, as compared to systemic corticosteroids alone.

In the second analysis, there were nine studies with a total of 1296 patients. No difference in the post-discharge relapse rate was demonstrated when inhaled corticosteroids alone were compared to systemic steroids alone. In both analyses, no differences in serious side effects were noted.

\section{Caveats}

While the methodologic quality of the trials was high and most were double-blind placebo controlled, the results of this Cochrane review were limited by the small sample size in most of the included studies. The included trials also demonstrated a high degree of heterogeneity. The authors of the Cochrane review recognized multiple potential causes for the heterogeneity, including differences in treatment protocols, populations, and study designs. Another cause of heterogeneity may have been the varied doses of inhaled corticosteroids used; one trial included in the review used a higher dose of inhaled corticosteroids and demonstrated better outcomes ${ }^{4}$.

Caution is also appropriate when drawing conclusions about the comparison of inhaled corticosteroids alone with systemic corticosteroids alone. Although inhaled corticosteroids and systemic corticosteroids had similar efficacy in prevention of asthma relapses, all of the patients in the included studies had mild to moderate asthma exacerbations. To assess severity, the included studies utilized either mean forced expiratory volume in one second (FEV1) or peak expiratory flow (PEF). For two of the adult studies, mild to moderate severity was defined as an FEV1 of $60 \%$ to $75 \%$ of predicted. The other adult study defined it as a PEF of $60 \%$ to $90 \%$ of predicted. The four pediatric studies used a PEF of $70 \%$ to $80 \%$ of predicted. In all of the studies, patients presenting with severe acute asthma were excluded. Thus, the findings cannot be generalized to patients with severe asthma exacerbations. Future studies could benefit from including cases of severe asthma exacerbation and analyzing effects by severity subgroups.

Only one of the studies was adequately powered to determine a difference or near-equivalency efficacy of inhaled corticosteroids and systemic corticosteroids, and it did not reach its targeted recruitment sample size ${ }^{4}$. Since inhaled corticosteroids may have fewer side effects than oral corticosteroids, further research should be specifically directed at determining the equivalence between the two routes of administration.

Finally, the studies in the Cochrane review did not include patients under the age of 12 , who comprise a large portion of the asthmatics who are treated in the emergency department. The results are thus insufficient to determine whether inhaled steroids should be prescribed to younger asthmatic patients after their discharge from the Emergency Department.

Most of the studies included in the systematic review grouped all patients with mild to moderate asthma together. Current outpatient management of asthma is based on a stepwise algorithm. As patients become more symptomatic and experience more frequent exacerbations, the treatment regimen is intensified. When asthma becomes better controlled, the medications and their doses are decreased accordingly. The goal of this approach is to control the patient's asthma while also limiting side effects. This step-wise approach includes medications such as short-acting beta agonists, inhaled corticosteroids, and systemic corticosteroids. Future studies that explore a wider range of asthma severity and include subgroup analyses may provide useful information about the role of inhaled corticosteroids in a variety of patients.

In conclusion, the current literature does not demonstrate a reduction in asthma relapse when inhaled steroids are added to oral corticosteroids. There is some evidence that high dose inhaled corticosteroids alone may be as effective as systemic corticosteroids, though current data is insufficient 
to show equivalency. Because of the limitations of the existing trials, we have assigned a color recommendation of Yellow (Unclear if benefits). Future larger trials enrolling a wider spectrum of disease severity and age may clarify what if any role exists for inhaled corticosteroid use after emergency department discharge.

\section{Competing interests}

No financial, legal or political competing interests with third parties (government, commercial, private foundation, etc.) were disclosed for any aspect of the submitted work (including but not limited to grants, data monitoring board, study design, manuscript preparation, statistical analysis, etc.).

\section{References}

1. Fergeson JE, Patel SS, Lockey RF. Acute asthma, prognosis, and treatment. Journal of Allergy and Clinical Immunology. 2017;139(2):438-447. doi: 10.1016/j.jaci.2016.06.054

2. Rowe BH, Kirkland SW, Vandermeer B, Campbell S, Newton A, Ducharme FM et al. Prioritizing Systemic Corticosteroid Treatments to Mitigate Relapse in Adults with Acute Asthma: A Systematic Review and Network Meta-analysis. Academic Emergency medicine. 2017;24(3)371-381. doi: 10.1111/ acem.13107

3. Abdullah AA. Corticosteroids in the treatment of acute asthma. Annuals of Thoracic Medicine. 2014;9(4):187-192. doi: $\underline{10.4103 / 1817-1737.140120}$

4. Edmonds ML, Milan SJ, Brenner BE, Camargo CA Jr, Rowe $\mathrm{BH}$. Inhaled steroids for acute asthma following emergency department discharge. Cochrane Database Syst Rev. 2012;12:CD002316. doi: 10.1002/14651858.CD002316.pub2

5. Edmonds ML, Camargo CA, Saunders LD, Brenner BE, Rowe $\mathrm{BH}$. Inhaled steroids in acute asthma following emergency department discharge. Cochrane Database Syst Rev. 2000;(3):CD002316. doi: 10.1002/14651858.CD002316 